\title{
investigación
}

\section{REVESTIMIENTOS INTERNOS CERAMICOS PARA CHIMENEAS INDUSTRIALES}

\section{(CERAMICS LINERS FOR INDUSTRIAL CHIMNEYS)}

\author{
Jesús Serradilla Echarri \\ Ingeniero Técnico de Obras Públicas
}

$(507-4)$

\author{
RESUMEN \\ En el presente articulo, se estudian los diversos factores que \\ intervienen en el estudio y posterior puesta en servicio de los \\ revestimientos internos cerámicos para chimeneas de uso industrial. \\ Se estudian inicialmente las diversas opciones existentes y \\ posterior decisión en cuanto a la solución a adoptar. \\ También se especifican las caracteristicas fisico-quimicas propias \\ del material, así como los criterios a seguir en cuanto al \\ dispositivo adecuado para el Control de Calidad del material.
}

\section{INTRODUCCION}

Al margen de la importancia del cálculo estático de una chimenea industrial, merece especial cuidado el estudio del revestimiento interno, debido a que en periodo de explotación soporta el contacto directo con los gases de evacuación.

Generalmente, los gases de evacuación llevan en su composición elementos nocivos que actuarán directa y profundamente sobre el material del revestimiento, por lo que en fase de proyecto se diseñará el mismo como resistente a los elementos físicos y químicos promovidos por los gases.

Son muchos los tipos de revestimientos que se pueden estudiar, aunque los subdividiremos en tres grandes grupos:

- Revestimientos metálicos.

- Revestimientos cerámicos.

- Revestimientos especiales (PVC, GRP...).

En el presente artículo, nos limitaremos al estudio de los revestimientos cerámicos; que en los últimos años en España han alcanzado un desarrollo elevado, debido sin duda a la aparición del concepto de gran chimenea, al aumentar las restricciones en materia de contaminación, que han afectado a las chimeneas de centrales térmicas ampliamente desarrolladas en el último Plan Energético Nacional.

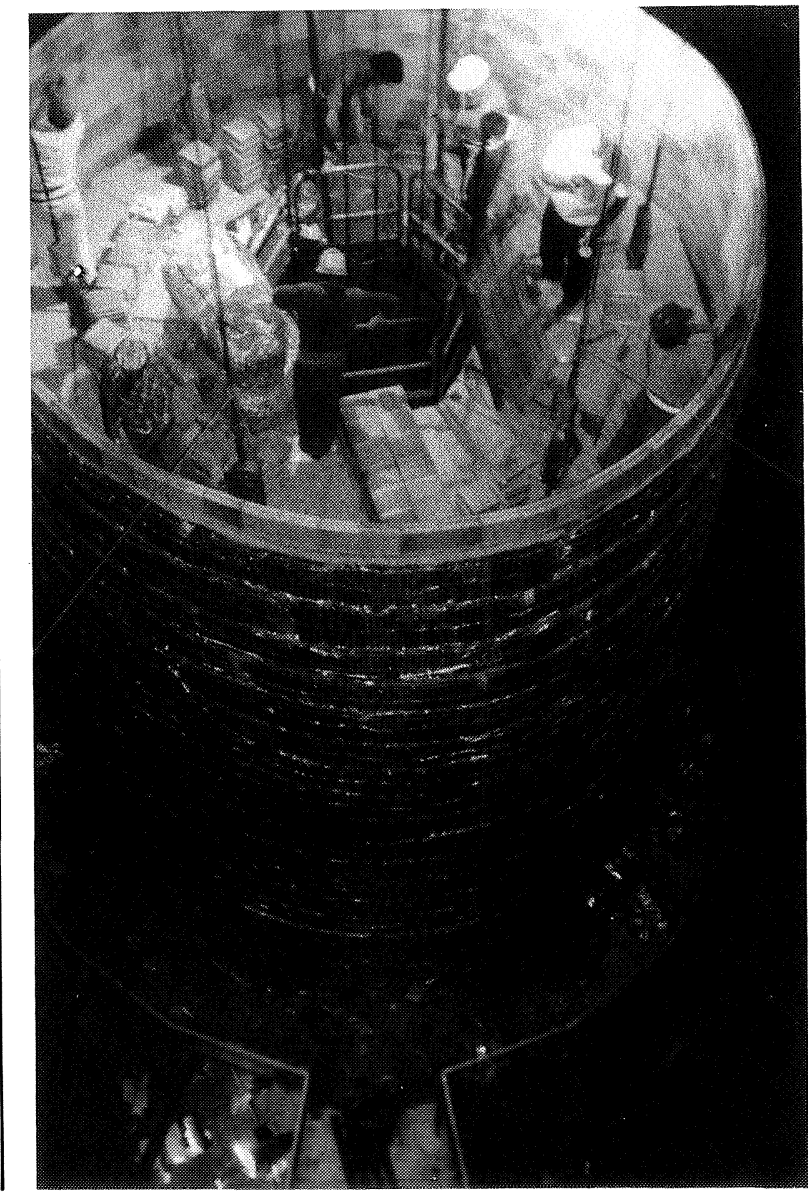




\section{CRITERIOS DE DISEÑO}

En el diseño del revestimiento interno de una chimenea, son tres los factores primordiales a tener en cuenta:

- Temperatura de funcionamiento.

- Ataque químico.

- Cambios bruscos de temperatura.

Aunque también se estudian de manera exhaustiva los siguientes:

- Concentración de productos químicos nocivos en los gases.

- Concentración de partículas sólidas en los gases.

- Velocidades máximas y mínimas de los gases.

- Presión del gas a la entrada de la chimenea.

- Temperatura ambiental.

- Efectos dinámicos externos (viento, sismo, soleamiento...).

Atendiendo al tipo de instalación al que se dedica la chimenea, puede tener carácter prioritario alguno de los factores citados anteriormente, aunque cada implantación requerirá un estudio minucioso de sus propias condiciones de explotación.

\section{DIMENSIONAMIENTO}

A continuación, vamos a seguir los diferentes pasos que se deben cubrir para llegar al dimensionamiento de una pared de revestimiento, atendiendo a los factores determinantes citados anteriormente.

- Supongamos una conducción de humos, que en régimen normal de trabajo la temperatura media y estable de los gases es de $200^{\circ} \mathrm{C}$, y que la temperatura ambiental exterior es de unos $25^{\circ} \mathrm{C}$, el estudio preliminar nos daría un revestimiento básico como el indicado en la figura 1.

- Aplicaremos la ecuación de aproximación de salto térmico:

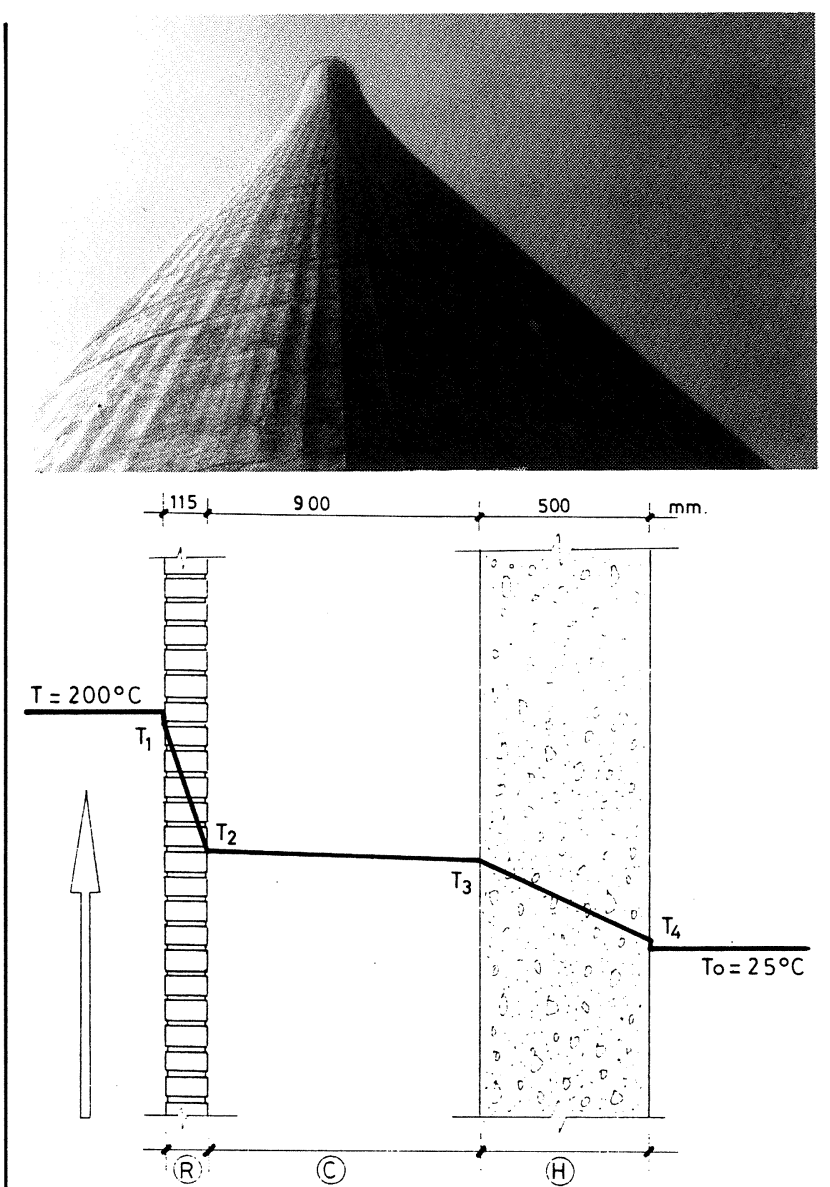

(R) REVESTIMIENTO Silico-ALUMINOSO

(C) CAMARA de aIRE ventILADA

(H) FUSTE DE HORMIGON ARMADO Fig. 1

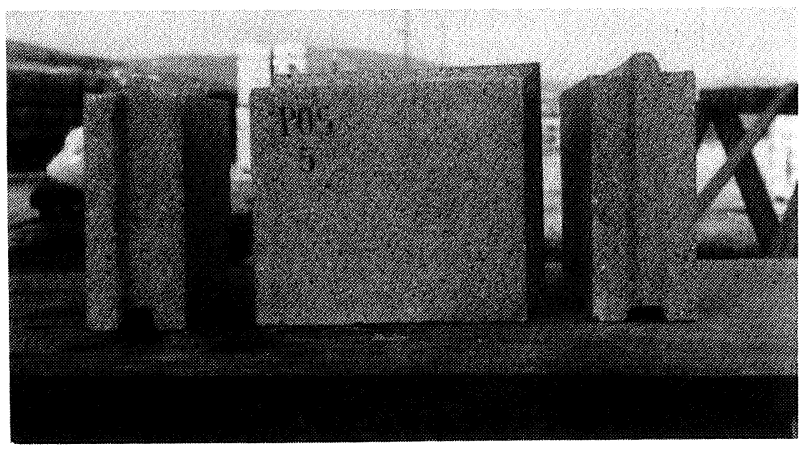

$$
\mathrm{T}_{\mathrm{x}}=\frac{\mathrm{t} \cdot \mathrm{D}_{\mathrm{bi}}}{\mathrm{C}_{\mathrm{c}} \cdot \mathrm{D}_{\mathrm{c}}}\left[\frac{\mathrm{T}-\mathrm{T}_{\mathrm{o}}}{\frac{1}{\mathrm{rq} \cdot \mathrm{K}_{1}}+\frac{\mathrm{t}_{\mathrm{b}} \cdot \mathrm{D}_{\mathrm{bi}}}{\mathrm{rq} \cdot \mathrm{C}_{\mathrm{b}} \cdot \mathrm{D}_{\mathrm{b}}}+\frac{\mathrm{D}_{\mathrm{bi}}}{\mathrm{K}_{\mathrm{s}} \cdot \mathrm{D}_{\mathrm{s}}}+\frac{\mathrm{t} \cdot \mathrm{D}_{\mathrm{bi}}}{\mathrm{C}_{\mathrm{c}} \cdot \mathrm{D}_{\mathrm{c}}}+\frac{\mathrm{D}_{\mathrm{bi}}}{\mathrm{K}_{2} \cdot \mathrm{D}_{\mathrm{co}}}+(\ldots)}\right]
$$

- Y resolveremos las igualdades de intercambio de calor:

$$
\begin{gathered}
\mathrm{rq} \cdot \mathrm{Q}=\mathrm{rq} \cdot \mathrm{K}_{1}\left(\mathrm{~T}-\mathrm{T}_{1}\right)=\frac{\mathrm{rq} \cdot \mathrm{C}_{\mathrm{b}} \cdot \mathrm{D}_{\mathrm{b}}}{\mathrm{t}_{\mathrm{b}} \cdot \mathrm{D}_{\mathrm{bi}}}\left(\mathrm{T}_{1}-\mathrm{T}_{2}\right)=\frac{\mathrm{K}_{\mathrm{s}} \cdot \mathrm{D}_{\mathrm{s}}}{\mathrm{D}_{\mathrm{bi}}}\left(\mathrm{T}_{2}-\mathrm{T}_{3}\right)=\frac{\mathrm{C}_{\mathrm{c}} \cdot \mathrm{D}_{\mathrm{c}}}{\mathrm{t} \cdot \mathrm{D}_{\mathrm{bi}}}\left(\mathrm{T}_{3}-\mathrm{T}_{4}\right)= \\
=\frac{\mathrm{K}_{2} \cdot \mathrm{D}_{\mathrm{co}}}{\mathrm{D}_{\mathrm{bi}}}\left(\mathrm{T}_{4}-\mathrm{T}_{\mathrm{o}}\right)
\end{gathered}
$$


teniendo en cuenta la definición de los siguientes valores:

- $\mathrm{T}_{\mathrm{x}}=\mathrm{T}_{3}-\mathrm{T}_{4}$

- $\mathrm{T}=$ Temperaturas en ${ }^{\circ} \mathrm{C}$

- $\mathrm{t}=$ Espesores en $\mathrm{m}$

- $\mathrm{C}=$ Coef. conductividad térmica en $\mathrm{kcal} / \mathrm{m} \mathrm{h}{ }^{\circ} \mathrm{C}$

- $\mathrm{D}=$ Diámetros en $\mathrm{m}$

- $\mathrm{rq}=$ Relación de transmisión térmica

- $\mathrm{K}=$ Coef. transmisión térmica en $\mathrm{kcal} / \mathrm{m}^{2} \mathrm{~h}{ }^{\circ} \mathrm{C}$

- Obtendremos: $\quad \mathrm{T}_{1}=190{ }^{\circ} \mathrm{C}$

$$
\begin{aligned}
& \mathrm{T}_{2}=96^{\circ} \mathrm{C} \\
& \mathrm{T}_{3}=89^{\circ} \mathrm{C} \\
& \mathrm{T}_{4}=27^{\circ} \mathrm{C}
\end{aligned}
$$

- De aquí deducimos que el revestimiento estudiado no es admisible por dos motivos fundamentales:

1. La temperatura $T_{3}$, de la cara interna de la pared de hormigón no debe sobrepasar nunca los $70{ }^{\circ} \mathrm{C}$, (E.H. 80), en nuestro caso es de $89{ }^{\circ} \mathrm{C}$.

2. La tensión térmica del revestimiento cerámico viene dada por la expresión:

$$
\sigma_{\mathrm{t}}=1 / 2 \cdot \mathrm{E} \cdot \alpha_{\mathrm{t}} \cdot \triangle_{\mathrm{t}}
$$

siendo:

$\mathrm{E}=$ Módulo de elasticidad del material en $\mathrm{kp} /$ $\mathrm{cm}^{2}$.

$\alpha_{\mathrm{t}}=$ Coef. dilatación térmica del material en ${ }^{\circ} \mathrm{C}^{-1}$.

$\triangle_{\mathrm{t}}=$ Gradiente térmico de la pared $\left(\mathrm{T}_{1}-\mathrm{T}_{2}\right)$.

Para considerarse admisible $\sigma_{\mathrm{t}}<25 \mathrm{kp} / \mathrm{cm}^{2}$, en nuestro caso $\sigma_{\mathrm{t}}=51 \mathrm{kp} / \mathrm{cm}^{2}$.

- Como primera medida a adoptar consideraremos un elemento de calorifugado que nos elimine la alta temperatura en el hormigón, para lo cual disponemos una capa de aislamiento térmico entre el material cerámico y el fuste de hormigón. Por facilidad de montaje y para mejorar las condiciones térmicas de la pared, la enclavamos adosada a la cara externa del material cerámico, tal y como se indica en la figura 2 .

- Aplicando las ecuaciones de salto térmico [I], y las igualdades de intercambio de calor [II], obtenemos:

$$
\begin{aligned}
& \mathrm{T}_{1}=196{ }^{\circ} \mathrm{C} \\
& \mathrm{T}_{2}=163^{\circ} \mathrm{C} \\
& \mathrm{T}_{\mathrm{A}}=52^{\circ} \mathrm{C} \\
& \mathrm{T}_{3}=48{ }^{\circ} \mathrm{C} \\
& \mathrm{T}_{4}=27^{\circ} \mathrm{C}
\end{aligned}
$$

- Donde observamos que la temperatura interior del hormigón es inferior a $70{ }^{\circ} \mathrm{C}$, y que el gradiente térmico del revestimiento es $\mathrm{T}_{1}-\mathrm{T}_{2}=33^{\circ} \mathrm{C}$, que produce una tensión térmica, según la ecuación [III], de $17 \mathrm{kp} / \mathrm{cm}^{2}$; por lo tanto podríamos dar por bueno el revestimiento estudiado.

- Ahora bien, podríamos mejorar sustancialmente el revestimiento deducido y disponerlo en condiciones más favorables, modificando ligeramente el estudio dimensional de la pared, tal como se indica en la figura 3 , donde se ha incrementado el espesor del aislamiento y por el contrario se disminuye, por supuesto dentro de normas establecidas, el espesor del material cerámico.

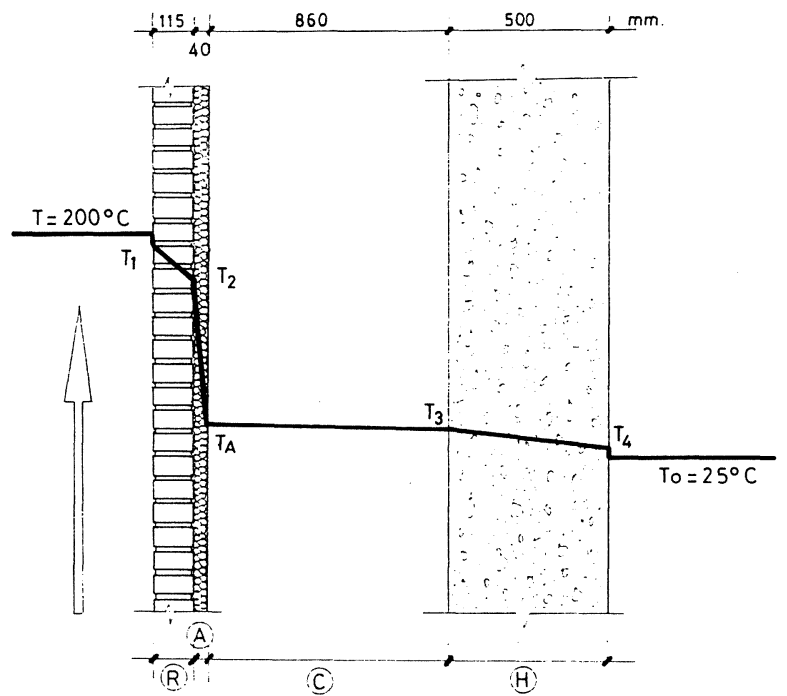

(R) REVESTIMIENTO SILICO-ALUMINOSO

(C) camara de aire ventilada

(H) FUSTE DE HORMIGON ARMADO

(A) aIslamiento termico

Fig. 2

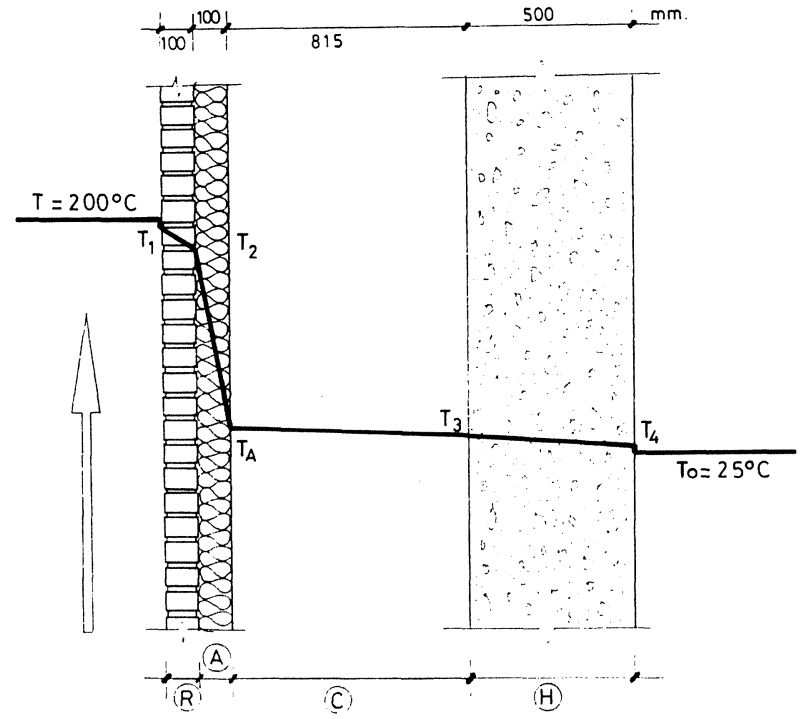

(R) REVESTIMIENTO SILICO.ALUMINOSO

(C) CAMARA DE AIRE VENTILADA

(H) FUSTE DE HORMIGON ARMADO

A. aIslamiento termico

Fig. 3 
- Aplicando las fórmulas [I] y [II], obtenemos:

$$
\begin{aligned}
& \mathrm{T}_{1}=193{ }^{\circ} \mathrm{C} \\
& \mathrm{T}_{2}=183^{\circ} \mathrm{C} \\
& \mathrm{T}_{\mathrm{A}}=42^{\circ} \mathrm{C} \\
& \mathrm{T}_{3}=38{ }^{\circ} \mathrm{C} \\
& \mathrm{T}_{4}=27^{\circ} \mathrm{C}
\end{aligned}
$$

- Obteniendo una temperatura en la cámara de aire que la hace accesible a personas y por lo tanto visitable. Por otro lado, el gradiente térmico de la pared de revestimiento es de $10^{\circ} \mathrm{C}$, que produce una tensión térmica de aproximadamente $5 \mathrm{kp} / \mathrm{cm}^{2}$, muy por debajo de la tensión admisible.

- En este momento podriamos dar por bueno el nuevo revestimiento optimizado estudiado, siempre que la instalación a la que sirve la chimenea funcione en régimen de temperaturas estables; es decir, que la temperatura de trabajo no fluctúe, y si lo hace sea mediante pequeñas oscilaciones térmicas realizadas de forma pausada.

- Ampliando el espectro real de posibilidades, vamos a suponer que la instalación a la que sirve la chimenea, debido a su funcionalidad, puede brúscamente variar la temperatura de régimen; por ejemplo hasta $400{ }^{\circ} \mathrm{C}$. El efecto se manifiesta de tal forma que la temperatura $T_{1}$ sube rápidamente mientras que $T_{2}$ no recupera de la misma forma, creándose en un breve espacio de tiempo un salto térmico superior a $150{ }^{\circ} \mathrm{C}$ entre ambas caras del material cerámico, superando ampliamente la tensión admisible del material. En un espacio de tiempo más prolongado, la curva de temperaturas se estabiliza sin más problemas; ahora bien, la reiteración de este fenómeno se suele manifestar inicial-

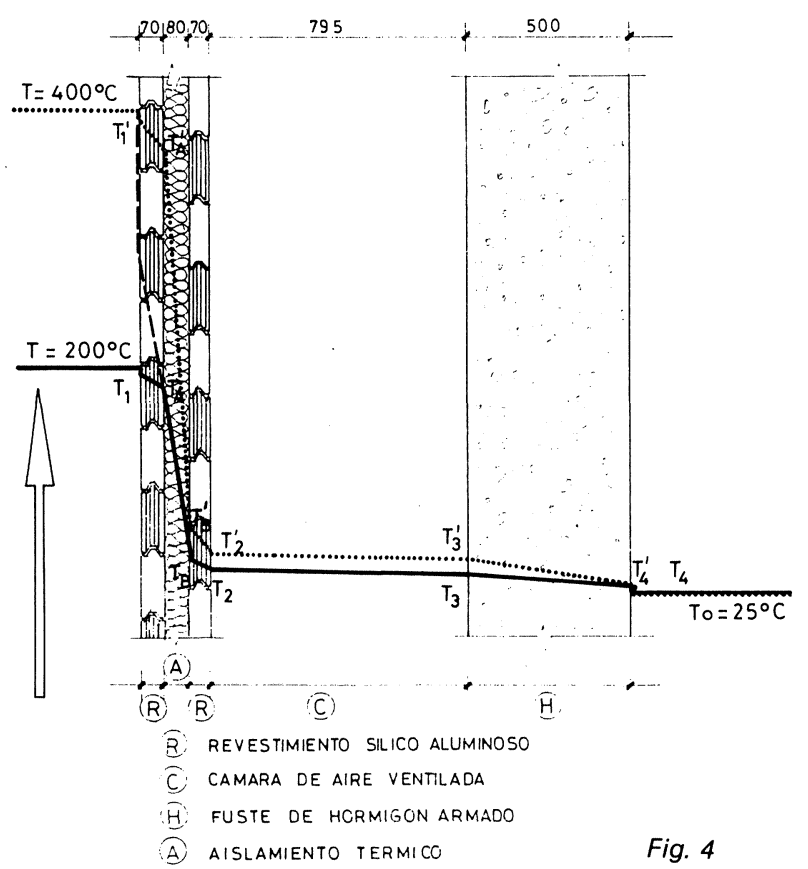

mente mediante pequeñas fisuras, que tienden a aumentar hasta incluso correr riesgos importantes.

- A primera vista podríamos deducir que la forma de combatir este problema seria la de reducir el espesor de la pared hasta la mínima expresión, con lo que se eliminaría el gradiente térmico y por lo tanto evitar los problemas debidos a tensiones internas del material.

- Obviamente, estariamos ante una solución óptima pero lógicamente inviable desde el punto de vista constructivo.

- Ahora bien, podriamos desarrollar una solución que tienda hacia esta utópica alternativa dentro de la viabilidad del cálculo estático. En la figura 4 se observa que esta solución consiste en un sistema de doble pared de material cerámico con una capa de aislamiento térmico intermedio, manteniendo la cámara de aire ventilada y visitable entre el conjunto de revestimiento y el fuste de hormigón.

- Cuando la temperatura de los gases sea de $200^{\circ} \mathrm{C}$, las temperaturas interiores serán:

$$
\begin{aligned}
& \mathrm{T}_{1}=198{ }^{\circ} \mathrm{C} \\
& \mathrm{T}_{2}=186^{\circ} \mathrm{C} \\
& \mathrm{T}_{\mathrm{A}}=51{ }^{\circ} \mathrm{C} \\
& \mathrm{T}_{\mathrm{B}}=40{ }^{\circ} \mathrm{C} \\
& \mathrm{T}_{3}=38{ }^{\circ} \mathrm{C} \\
& \mathrm{T}_{4}=27^{\circ} \mathrm{C}
\end{aligned}
$$

- Donde observamos que las dos paredes de material cerámico mantienen unos gradientes térmicos de baja cuantía, que no deben dar problemas de tensiones internas.

- Supongamos que, como en el caso anterior, los gases suben brúscamente de temperatura hasta $400{ }^{\circ} \mathrm{C}$, inmediatamente tendremos que $\mathrm{T}_{1}^{\prime}=300^{\circ} \mathrm{C}$ (aprox.), pero $T_{2}$ continúa en prácticamente $186^{\circ} \mathrm{C}$, en un pequeño espacio de tiempo se estabilizan, quedando de la siguiente forma:

$$
\begin{aligned}
& \mathrm{T}_{1}^{\prime}=395^{\circ} \mathrm{C} \\
& \mathrm{T}_{2}^{\prime}=369^{\circ} \mathrm{C} \\
& \mathrm{T}_{\mathrm{A}}^{\prime}=75^{\circ} \mathrm{C} \\
& \mathrm{T}_{\mathrm{B}}^{\prime}=53^{\circ} \mathrm{C} \\
& \mathrm{T}_{3}^{\prime}=50^{\circ} \mathrm{C} \\
& \mathrm{T}_{4}^{\prime}=27^{\circ} \mathrm{C}
\end{aligned}
$$

- El que en un pequeño espacio de tiempo el revestimiento esté trabajando por encima de la tensión admisible, normalmente no produce problemas importantes, debido a que por el pequeño espesor de pared la estabilización de las temperaturas se produce rápidamente, y que en la práctica es muy dificil que se dé el 
cambio brusco de forma casi instantánea, sino que por el contrario se realiza de forma gradual, por lo que los gradientes no serán tan extremos como en el ejemplo.

- Más adelante veremos que los condicionamientos a exigir al material nos permitirán más flexibilidad a la hora de juzgar el comportamiento del material de revestimiento ante los supuestos de choque térmico.

\section{PROPIEDADES FISICO-QUIMICAS}

- En un segundo paso en el estudio, trataremos de llegar a las condiciones físico-químicas que se deben exigir al material para ser adecuado para la instalación a la que va destinada.

- La norma DIN 1058 en su Capítulo 5.1.2, nos marca la pauta a seguir indicando que para gases de escape normalmente húmedos como en el caso que nos ocu$\mathrm{pa}$, al material se le deben exigir las siguientes propiedades:

- Densidad aparente (mínima)..... .

$1,9 \mathrm{t} / \mathrm{m}^{3}$

- Resistencia a compresión en frío (minima)................ $400 \mathrm{kp} / \mathrm{cm}^{2}$

- Absorción de agua (máxima) .... $10 \%$ en peso

- Solubilidad en ácido sulfúrico (máxima $\ldots \ldots \ldots \ldots \ldots \ldots \ldots$ 2,5 \% en peso

- Con el fin de completar los requerimientos necesarios para obtener un revestimiento homogéneo, de entrada deben limitarse los máximos de los dos primeros conceptos, de tal forma que se evite la posibilidad de que dos piezas contiguas sean muy dispares en sus calidades; pongamos por caso una pieza con una resistencia a compresión de $400 \mathrm{kp} / \mathrm{cm}^{2}$, y la siguiente con $1.000 \mathrm{kp} / \mathrm{cm}^{2}$, lógicamente el comportamiento térmico de ambas es muy diferente provocando tensiones complementarias de dificil control.

- Asimismo, y con el fin de delimitar éstas y otras propiedades del material, la experiencia obliga a especificar otras limitaciones a cumplir por el fabricante, estas son:

- Densidad aparente (máxima) ....

$2,1 \mathrm{t} / \mathrm{m}^{3}$

- Resistencia a compresión en frío (máxima) .................

- Contenido en $\mathrm{Al}_{2} \mathrm{O}_{3}+\mathrm{T}_{\mathrm{i}} \mathrm{O}_{2} \ldots \ldots$.

- Contenido en $\mathrm{Fe}_{2} \mathrm{O}_{3}$ (máximo) ... $700 \mathrm{kp} / \mathrm{cm}^{2}$ $33-36 \%$ $2,5 \%$

- Resistencia refractaria bajo carga cte. (Ta).................

- Resistencia piroscópica (Cono Seger) ................

- Resistencia a choques términos (mínima) .................

\section{MATERIALES DE UNION}

Tan importante como una buena elección de los materiales base del revestimiento, es el elegir un tipo de mortero o mástico de unión de piezas que cumpla perfectamente con la solicitación a que va a ser sometido, por ello debe cuidarse de que la mezcla a utilizar sea compatible con todos los factores que intervengan.

Dado que nos encontramos con instalaciones en las que es previsible la presencia de ácidos - bien por trabajar a baja temperatura por procesos de recuperación calorifica, bien porque el contenido de derivados del azufre en los humos sean importantes- se debe considerar un mástico antiácido, cuya base de fabricación sea vidrio soluble.

Es importante señalar que si en el mástico hay presencia de sodio, el ácido sulfúrico que se condensa reacciona con él para formar sulfato sódico, material hidratante que cristaliza en la unión de las piezas, captando la humedad existente y aumentando con el tiempo su volumen hasta casi un $50 \%$. Esta anomalia llega a producir tensiones internas muy importantes, hasta sobrepasar los $500 \mathrm{kp} / \mathrm{cm}^{2}$, que invariablemente producirán graves daños y por consiguiente grandes y costosas reparaciones.

Para evitar esto, se deberá utilizar un silicato potásico de 42,5 ${ }^{\circ}$ Bé, que aunque es de un costo inicial más elevado, es obvio que a la larga es infinitamente más rentable.

Con esto obtenemos no sólo un mástico de un comportamiento antiácido asegurado, sino que además conseguimos que las propiedades fisico-quimicas se asemejen a las del material a unir, con lo que la homogeneidad de la pared está garantizada, mejorando el punto débil que supone en todo revestimiento la junta de unión.

\section{OTROS FACTORES}

Hasta ahora hemos visto las diferentes propiedades que deben cumplir los materiales a utilizar en un correcto revestimiento cerámico para el tipo de estructura que nos ocupa; vamos a estudiar a continuación otros condicionamientos a tener en cuenta y que son de importancia a la hora de evaluar el funcionamiento futuro de la instalación.

En chimeneas que funcionan en régimen de tiro natural, es lógico pensar que existen pérdidas de tiro debidas al rozamiento entre la superficie interior del conducto cerámico y los gases; estas pérdidas de tiro son más importantes cuanto más rugosa e imperfecta es la pared de revestimiento.

Antiguamente la construcción se realizaba mediante los $1.300{ }^{\circ} \mathrm{C}$ clásicos ladrillos de forma paralelepipédica, unidos mediante mortero formado por áridos silíceos y cementos aluminosos; esta conjunción constituia un revestimiento con aristas vivas, las secciones horizontales eran de forma poligonal y las juntas de unión eran de una intensidad 10 ciclos de un mínimo de $10 \mathrm{~mm}$ ampliándose hacia el exterior. 
El primer paso en la mejora de los revestimientos se consiguió sustituyendo las formas rectangulares de los ladrillos por piezas en forma de cuñas trapezoidales, con lo cual se disminuian los cantos vivos y se uniformizaba el espesor de las juntas de mortero en su sentido radial; ahora bien, la sección horizontal seguía siendo de forma poligonal y la junta de mortero continuaba siendo de 10 mm o más.

El siguiente paso fue una modificación del anterior, mediante el cual el formato del ladrillo por su cara interna era dotada de una curvatura aproximada a la real, con lo cual se conseguía eliminar de forma casi total las aristas vivas y se formaba una superficie más uniforme.

Estos revestimientos presentaban la dificultad de su elevado espesor frente a problemas producidos por choque térmico en fluctuaciones de temperatura de régimen, ante lo cual se desarrollaron los sistemas constructivos actuales, en los que las piezas cerámicas se fabrican en forma de dovelas machihembradas en sus cuatro caras de contacto, lo cual conjuntamente con los másticos de vidrio soluble descritos anteriormente, que permiten huellas de 1 ó $2 \mathrm{~mm}$, se dispone de una construcción adecuadamente curvada y prácticamente continua, donde el rozamiento queda muy disminuido y por lo tanto las pérdidas de tiro seriamente combatidas; asimismo este tipo de construcción permite una mejor trabazón de piezas y por lo tanto acepta menores espesores garantizando la estabilidad del conjunto y mejorando sus condiciones frente al choque térmico producido por cambios bruscos en la temperatura.

Por otro lado, cuando se va a estudiar un revestimiento para una chimenea, se parte de la base de construcción del revestimiento por escalones; es decir, por tramos independientes de determinada altura apoyados en el propio fuste de hormigón, aunque en casos especiales se construye el revestimiento indendientemente del fuste de hormigón, de forma monolítica y apoyado sobre la losa de cimentación.

La construcción por escalones o trómeles, permite por un lado aumentar la estabilidad de la estructura debido al incremento de la componente de peso propio, y por otro lado, la construcción de una especie de sistema articulado que admita los consiguientes movimientos del conjunto debidos a factores externos como son: viento, sismo, soleamiento, etc.

La longitud de cada trómel es muy variable pudiendo comprender una amplia gama entre los 9 y $25 \mathrm{~m}$, incluso en alturas superiores para casos especiales. Para determinar la altura concreta en cualquier caso habrá que tener en cuenta factores como: grado sísmico, componente de viento, resistencia a compresión en frío del material y coeficientes de dilatación térmica.

Este tipo de construcción crea unas zonas de tratamiento especial como son las uniones entre trómeles, pero que se solucionan dando continuidad al conjunto con previsión de juntas de dilatación, así como piezas vierte-aguas que preservan la cámara interior de posibles filtraciones.

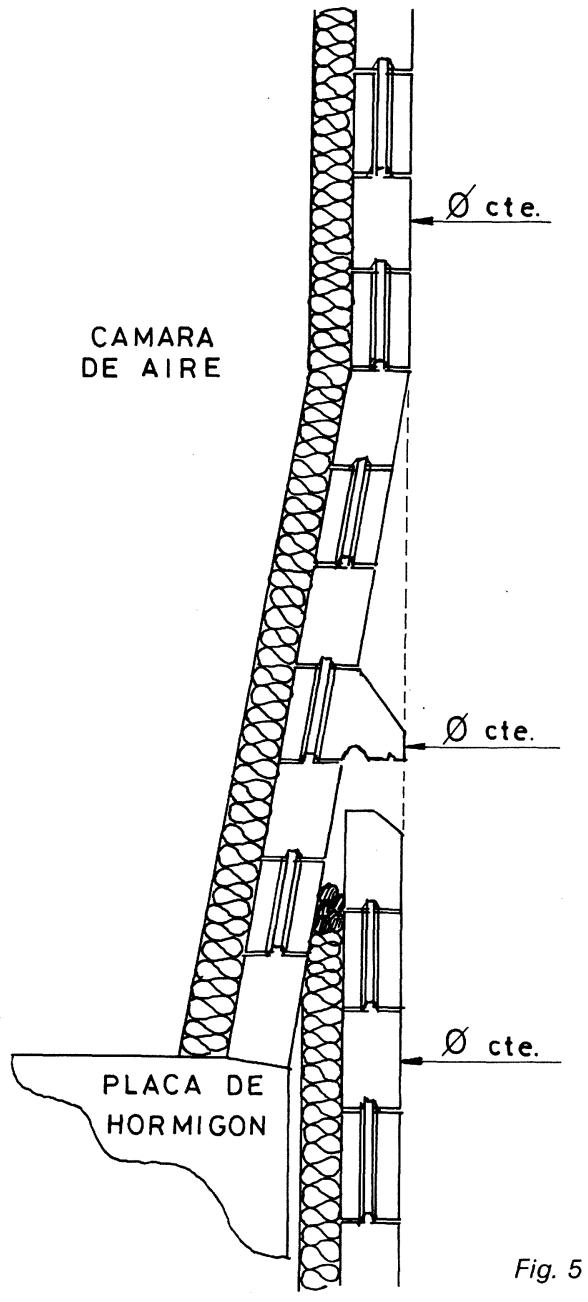

En la figura 5 se da una muestra de cómo puede ser resuelto el diseño.

Una zona de especial tratamiento es la coronación de chimeneas, ya que es el punto de contacto entre los humos calientes evacuados y la atmósfera, y por lo tanto donde el ataque ácido se manifiesta en su mayor intensidad.

Las soluciones a adoptar son muy variadas, pues son comúnmente utilizados anillos de fundición, anillos de acero inoxidable así como cubrimientos de material antiácido. Para instalaciones donde es previsible un importante ataque ácido se recomienda la tercera, ya que es la que mayor garantía ofrece en el transcurso de su período de explotación.

\section{CONTROL DE CALIDAD}

Es importante señalar que cualquier diseño de revestimiento debe acompañarse de un riguroso estudio de las calidades del material, con el que puedan garantizarse los condicionamientos exigibles al conjunto a construir.

No existe una norma única a la que poder recurrir para la verificación completa de una fabricación concreta; ahora 
bien, después de varios años de estudios, estadísticas y comprobaciones, se han desarrollado los procedimientos adecuados a seguir para estar en disposición de evaluar las cualidades del material.

En principio, se realizarán dos tipos de controles:

- Control visual y dimensional.

- Control de ensayos de calidad.

\section{Control visual y dimensional}

Inicialmente deberá realizarse una planificación de las visitas de inspección, de acuerdo con los programas de fabricación. Todo el conjunto se subdividirá en lotes independientes de 10.000 piezas aproximadamente cada uno, procedentes de un periodo concreto de fabricación y de una misma posición. Las posiciones de menos de 10.000 Uds., formarán lotes de 3.000 piezas.

Los palets correspondientes deben ser marcados y numerados por el inspector. De todo el conjunto se extraen un determinado número de palets, y una vez separados del resto, se extrae un determinado número de piezas de cada uno.

La determinación de número de palets y número de piezas puede realizarse de acuerdo con la norma UNE 61003-75 (Apartado 5.1).

Una vez separadas las piezas a estudiar, se realizan visualmente los siguientes controles:

- Indice de cocción.

- Resistencia de aristas.

- Existencia de fisuración y grietas.

- Prueba de sonido.

- Comprobación de dimensiones.

Los criterios de aceptación y rechazo pueden regirse por las normas UNE 61-005-75 y UNE 61-006-75.

Todos los resultados obtenidos deben reflejarse en impresos idóneos y archivados en el Dossier General de Control de Calidad. Al final del artículo se incluye el impreso ENSAYOS 1 como impreso tipo.

\section{Control de Ensayos de Calidad}

Este tipo de control se refiere a la verificación de las propiedades físico-químicas del material y se realiza de la siguiente forma:

En el transcurso de la inspección para control visual y dimensional, de cada lote elegido, se realiza un muestreo de piezas con unos criterios previamente establecidos. Las muestras extraídas son numeradas y referenciadas en un impreso como puede ser el de RECEPCION DE MUESTRAS que se incluye al final del articulo, y se en- vian las piezas al laboratorio homologado que previamente se designe para la realización de las pruebas. Los ensayos básicos a realizar son los siguientes:

- Análisis químico (DIN 51061).

- Densidad aparente (UNE 61-033-75).

- Absorción de agua (UNE 61-033-75).

- Porosidad aparente (UNE 61-033-75).

- Resistencia a compresión en frío (DIN 28062).

- Solubilidad en ácido (DIN 51102).

- Resistencia a choque térmico (UNE PNE 61041).

- Resistencia refractaria bajo carga cte. (Ta).

- Resistencia piroscópica (Proc. Cono SEGER).

Las normas indicadas son orientativas, permitiéndose utilizar otras según el criterio del inspector. Los resultados deben reflejarse en impresos adecuados; un caso puede ser la hoja que se adjunta al final del artículo como ENSAYOS 2. Existe en diversas normas el criterio de considerar conveniente que las muestras extraídas sean divididas en tres partes, ensayando únicamente una de ellas y quedando las otras dos en depósito, ante la posibilidad de resultados dudosos para la realización de ensayos complementarios. Ahora bien, una forma de simplificar el tema es no proceder a la división de las muestras con lo que no se reservarian probetas para posibles contraensayos, esto en principio nos permite realizar más ensayos sobre las muestras extraídas y no supone problemas en caso de ensayos defectuosos, ya que se puede acudir al lote y palet de origen con el fin de realizar nuevo redesmuestre de piezas.

Por este motivo adquiere mayor importancia, como se ha indicado anteriormente, la mayor homogeneidad de los lotes y, sobre todo, que la procedencia de ellos coincida con períodos de fabricación comunes.

Hay un capitulo que merece la pena resaltar por su importancia, y es que durante el periodo de control es conveniente que el inspector obtenga los resultados de los ensayos que por rutina realice el fabricante del material en su propio laboratorio, localizándolos por fechas de fabricación, esto se tratará sólo como documentación informativa, sin valor oficial alguno, pero puede suministrar una información muy interesante; primero porque si el grado de calidad alcanzado en este autocontrol se compara con el obtenido en la inspección oficial, nos dará un indice de fiabilidad del fabricante y por otro lado, podemos disponer de forma rápida de una información aproximada sobre la calidad del material o detectar alguna anomalia en la fabricación, cosa que no podriamos realizar si sólo disponemos de los informes del laboratorio oficial, cuya información, por regla general, se demora lo suficiente como para eliminar nuestra capacidad de reacción ante este tipo de eventualidades.

Una vez cumplimentada toda la información sobre los resultados, se debe reunir conjuntamente con los planos de despiece del material, con los informes de las visitas 


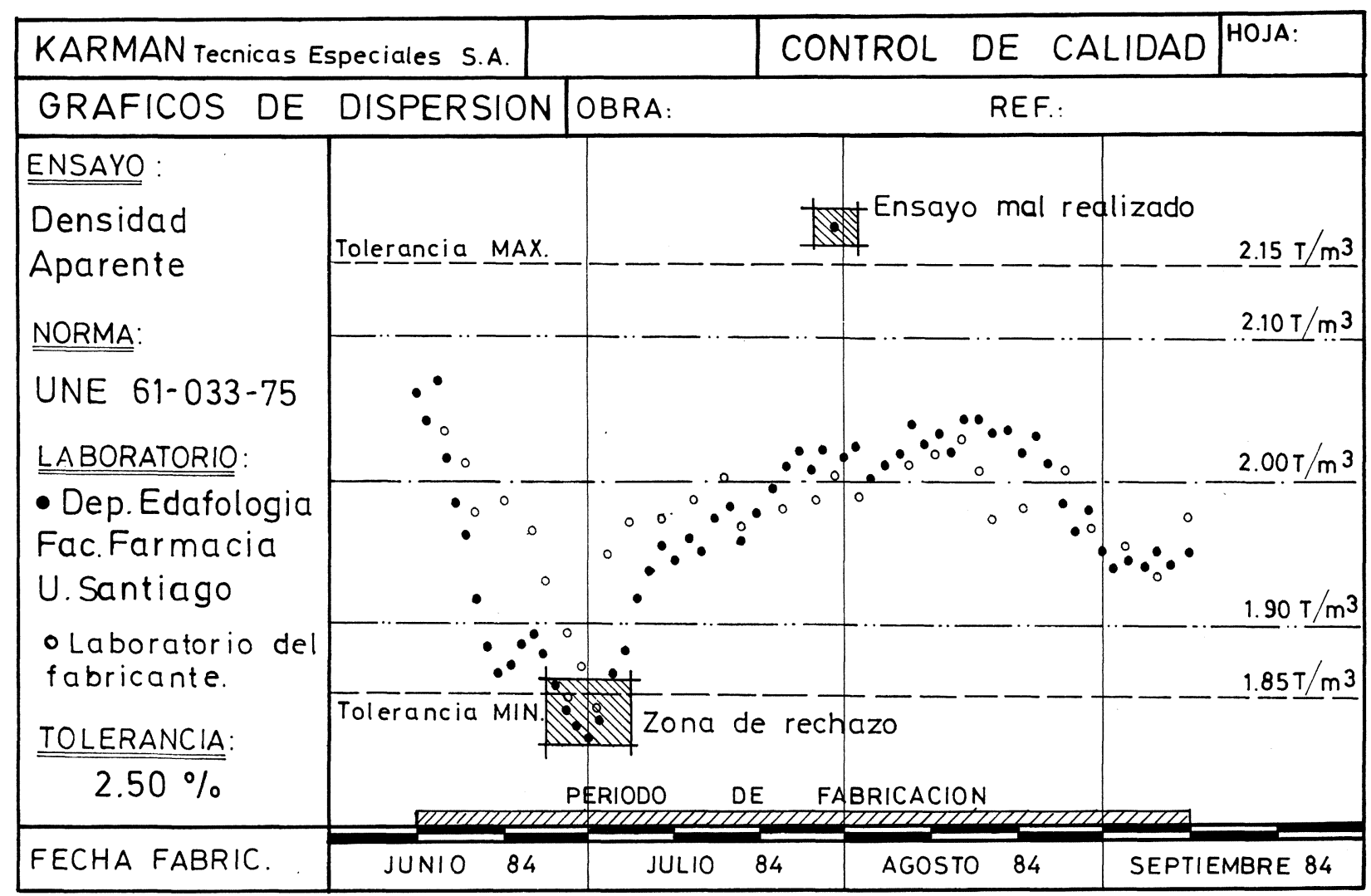

Fig. 6

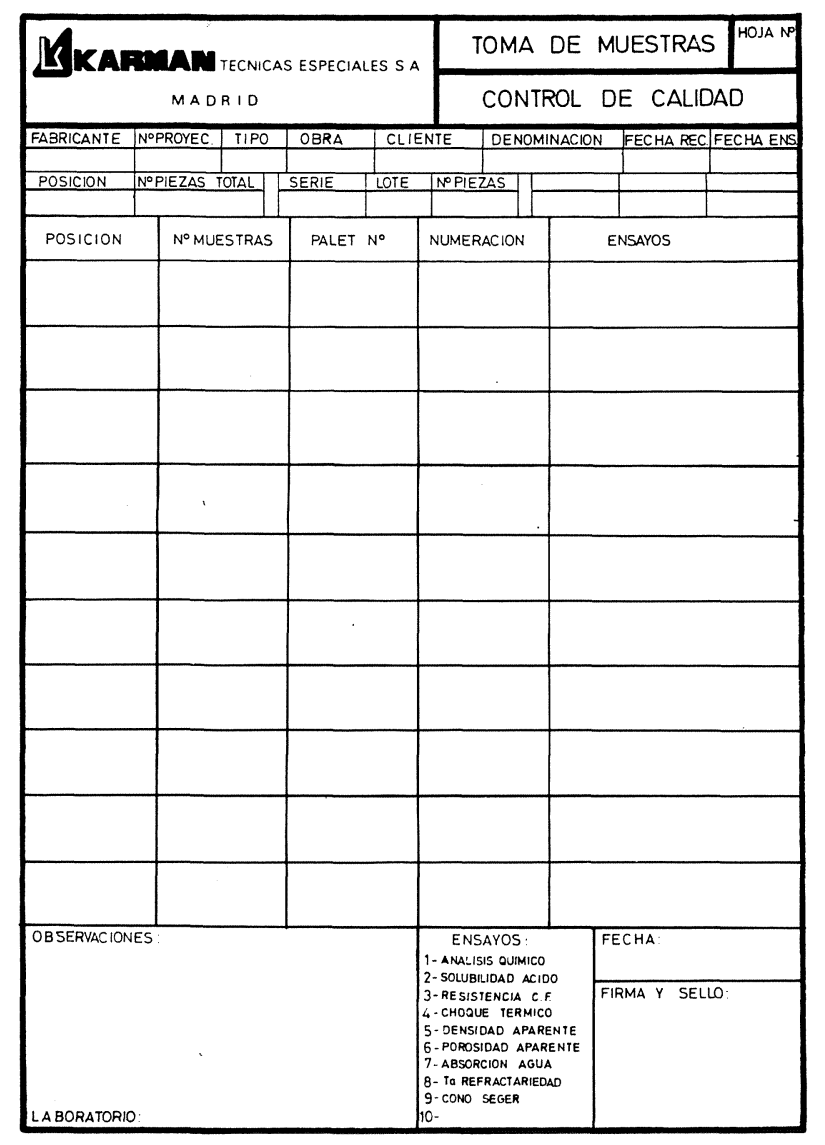

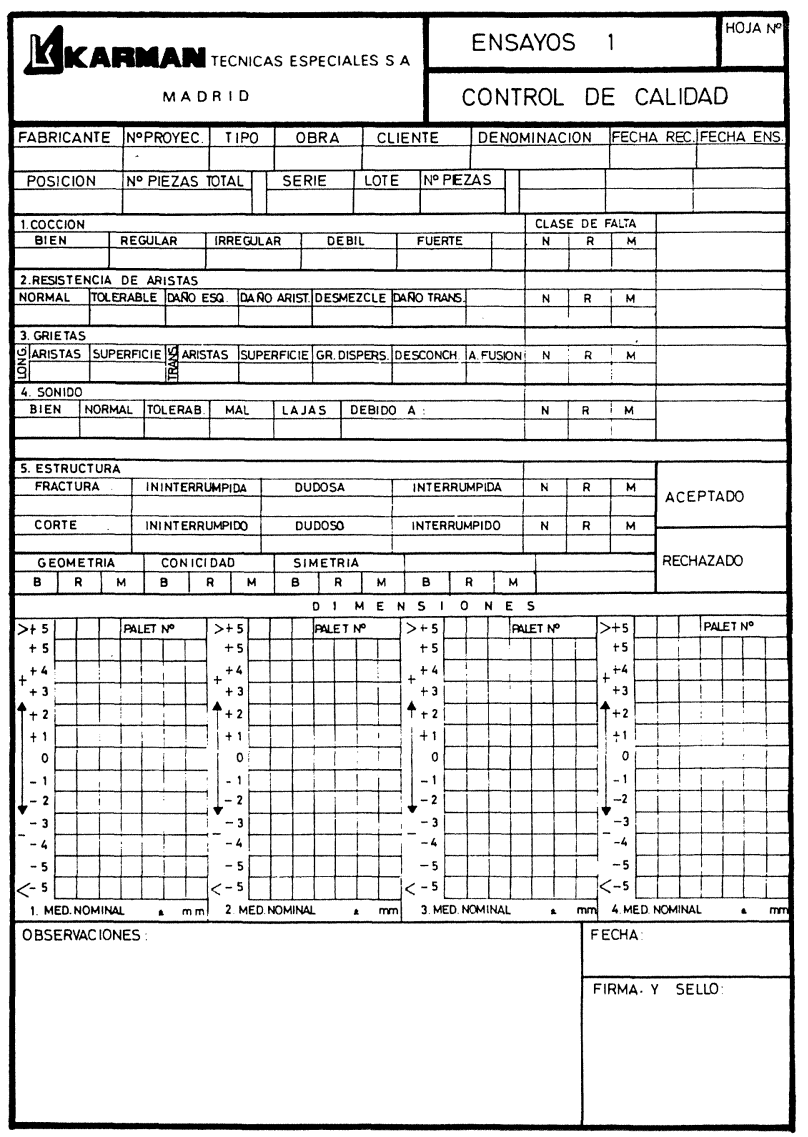




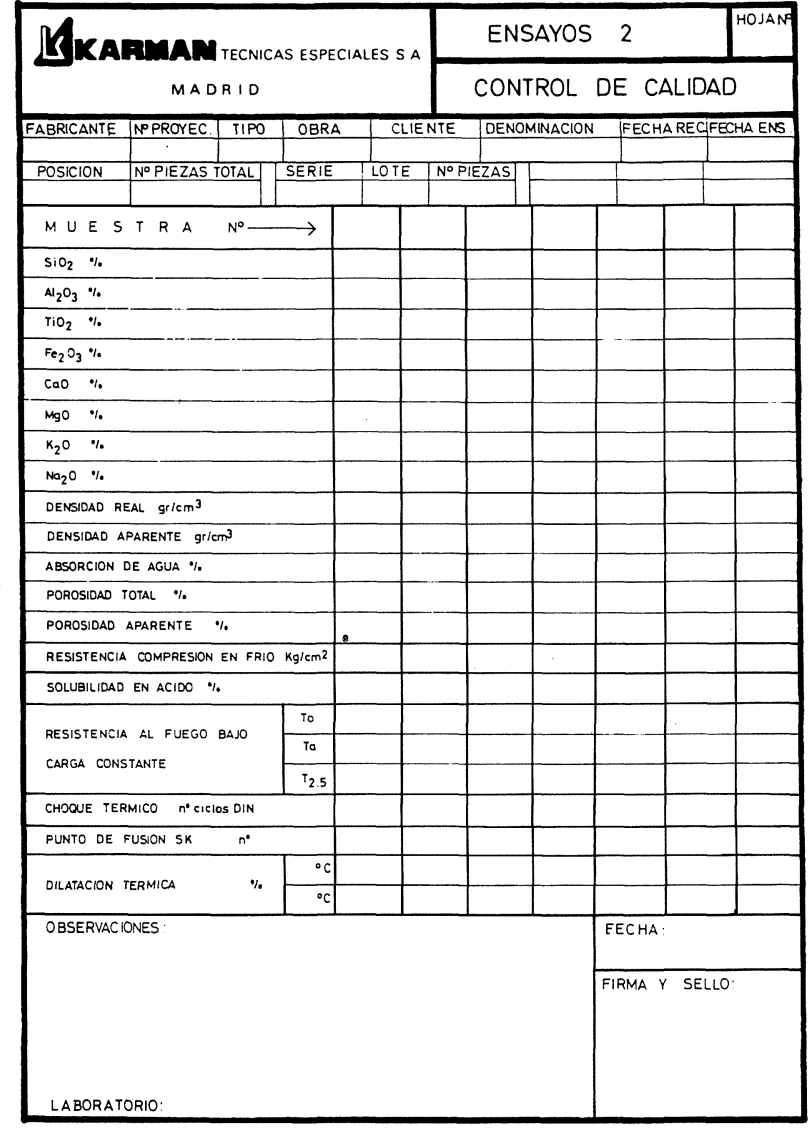

de inspección y con los informes de los laboratorios homologados e inlcuirlo en el Dossier General de Control de Calidad de la obra.

Con el fin de dar una idea de conjunto, es conveniente la realización de resúmenes que a simple vista nos mues- tren la calidad del conjunto, por ello es recomendable la realización de gráficos como en el caso hipotético de la figura 6 , y de resúmenes globales que, a posteriori, podamos consultar de forma rápida y eficaz.

Respecto al Control de Calidad del mortero, es aplicable todo lo citado en este apartado, teniendo la precaución de que previamente a la construcción se realicen un conjunto de probetas DIN (cilindros de $50 \mathrm{~mm}$ de altura por $50 \mathrm{~mm}$ de diámetro), sobre las que ensayar.

\section{CONSIDERACINES FINALES}

En este artículo hemos tratado de explicar un conjunto de consideraciones y pautas a seguir, que nos han sido muy eficaces durante los últimos años en las chimeneas construidas por nosotros; ahora bien, ni que decir tiene que existen otras formas de tratamiento del tema, ya que, como citamos antes, no existe una normativa específica sobre el tema que pueda adoptarse de forma generalizada.

La relación de chimeneas controladas por el procedimiento descrito durante los últimos años son las siguientes:

C. T. TERUEL (Andorra)........ $343 \mathrm{~m} \quad 2.816 \mathrm{t}$

C. T. COMPOSTILLA (León)...... $270 \mathrm{~m} \quad 1.885 \mathrm{t}$

REF. ERT (Huelva) ........... $140 \mathrm{~m} \quad 380 \mathrm{t}$

C. V. Jinamar (Las Palmas)........ $160 \mathrm{~m} \quad 600 \mathrm{t}$

PLA FLOAT (Venezuela) ......... $70 \mathrm{~m} .151 \mathrm{t}$

C. T. ITABO (Rep. Dominicana).... $70 \mathrm{~m} \quad 250 \mathrm{t}$

C. T. SOTO RIBERA (Oviedo) .... $\quad 200 \mathrm{~m} \quad 520 \mathrm{t}$

CEL. SOPORCEL (Portugal)...... $90 \mathrm{~m} \quad 260 \mathrm{t}$

C. T. LOS BARRIOS (Cádiz) ..... $230 \mathrm{~m} \quad 911 \mathrm{t}$

C. T. SABON Recons (La Coruña)... $200 \mathrm{~m} \quad 467 \mathrm{t}$

$$
\text { is 论弥 }
$$

